\title{
Obesity Epidemic: A Global Perspective
}

\author{
Gundu HR Rao* \\ Laboratory Medicine and Pathology Director, University of Minnesota, USA
}

*Corresponding author: Gundu HR Rao, Laboratory Medicine and Pathology Director, Thrombosis Research, Lillehei Heart Institute, University of Minnesota, USA

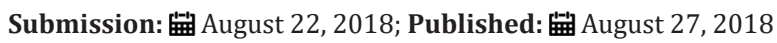

\section{Editorial}

Excess weight, body-mass index (BMI), and obesity, have become global epidemics. According to the experts, if post-2000 trends continue, the probability of meeting the global obesity target is virtually zero [1]. Several recent studies have assessed the national, regional and global, prevalence of excess weight and obesity [2-10]. Influence of modifiable risk factors, such as blood pressure, serum cholesterol, fasting glucose, and body mass index on cardiovascular disease (CVD) is well established. Each metabolic risk factor is robustly related to the progress of CVD. Global estimates indicate, considerable potential for CVD reduction, with population-wide lowering of BMI [2]. A bilateral study, between the researchers at the Madras Diabetes Research Foundation (MDRF), and the staff at the University of Minnesota, found that compared to the US, the waist-weight ratio was significantly higher, in men and women from India [3]. These results support the hypothesis, that South Asians are particularly predisposed toward central adiposity. There is a great need to assess the role of BMI, waist circumference, and waist-to-hip ratios (WHR), to see if these parameters, singly or in combination, improve CVD risk prediction, so that ethnic-specific or region-specific guidelines, could be developed for prevention strategies [5]. Chinese researchers have reported, significant association with increased WHR, with a stronger association among women [11]. Global epidemics of metabolic diseases, if not controlled soon, could cause economic disaster in many countries. A recent (2018), National Health Service (NHS), UK model indicates, that NHS Health Check programme is contributing significantly, to the improvements in health and reducing healthcare inequalities. They predict that feasible changes in the organization of the programme, could result in more than 3 -fold increase in health benefits [12]. In an earlier article in this journal, we articulated the management of excess weight and obesity [13]. In this article, we will discuss three possible interventions, which may reduce or reverse the metabolic risks, associated with excess weight and obesity.

Major national, regional, and global reports on obesity, have provided us an extensive analytical data, on the incidence and prevalence of excess weight and obesity worldwide. Despite the fact, these diseases pose a great global economic burden, these reports have not come up with any easy solutions, to reduce or reverse the trend in the increase of these diseases. Excess weight and obesity are associated, with increased risk for the development of type- 2 diabetes, and cardiovascular diseases. In view of these findings, there is a great interest in weight loss programs, that may alleviate this metabolic disease. It is hard to lose weight, but it is much harder to maintain the weight loss or sustain a healthy weight. A meta-analysis of 13 randomized trials suggested, that people on ketogenic diets, tend to lose more weight and keep more of it off, than people on low-fat diets. On the other hand, there are some reports, suggesting that ketogenic diet may predispose individuals to insulin resistance and diabetes. Professor Roy Taylor of Newcastle University, discussing the etiology of diabetes and reversibility, stated, that the first hint that type -2 diabetes is a fully reversible syndrome, came from bariatric surgery [14]. Based on his studies with low calorie diet, Roy Taylor reports, "Type-2 diabetes has long been regarded as inevitably progressive, requiring increasing number of oral hypoglycemic agents and eventually insulin, but is now certain, that the disease process can be halted with restoration of normal carbohydrate and fat metabolism. Type 2 diabetes can be understood as potentially reversible metabolic state precipitated by the single cause of chronic excess of intra organ fat". This hypothesis is worth further exploration, at the individual as well as population level.

Since we are discussing possible interventions, to reduce or reverse obesity related complications, we should consider the fact, that over 20 million children are born with low birth weight worldwide, and these children are "at risk" for developing metabolic diseases $[15,16]$. A study by the Harvard School of Public Health, has demonstrated that a multi-nutrient supplement for pregnant women, may alleviate this fetal origin of metabolic risk [17]. According to a Centers for Disease Control (CDC) and prevention report (June 2018), the prevalence of childhood obesity in the USA was $18.5 \%$ (14 million) and it also demonstrated a significant ethnic difference (Hispanic 25.8\%, African Americans 22\%). According to a WHO report, in 2010, 43 million children were overweight, and 98 million were at risk for overweight. This trend is estimated to double by 2020. According to Minnesota researchers even in 
children, oxidative stress and adipokine levels worsen throughout the continuum of obesity. Kelly and associates in Minnesota, conducted a study to assess subclinical inflammation, fasting insulin, and endothelial dysfunction, before and after exercise in overweight children. These researchers demonstrated, that just eight weeks of moderate exercise, increased fitness, normalized endothelial function and lowered inflammation [18].

Obesity in general, is also characterized by chronic low- grade inflammation, with permanently increased oxidative stress [19] Over expression of oxidative stress, damages cellular structures by generating excess superoxide, and lowers antioxidant mechanisms, leading to the development of obesity related clinical complications. Increased generation of superoxide in the vessel wall, lowers the production of nitric oxide, and creates an imbalance in the vasoactive compounds. This imbalance between the vasodilators and vasoconstrictors, will lead to the development of endothelial dysfunction, which we consider is the earliest sign of vascular disease. The transcription factor Nrf-2 (nuclear factor, erythroid-2-related factor-2, Nrf-2) for instance, is a master regulator of 500 genes (survival genes). Recent studies have demonstrated that induction of Nrf-2 and Ho-1 by Protandim (a mixture of five phytochemicals; Ashwagandha, Bacopa, Green tea, China Milk Thistle and Turmeric), is associated with a reduction of oxidative stress. Studies by Joe M McCord and associates, on the effect of Protandim on various pathways have shown, significant modulation, not only of pathways involving antioxidant enzymes, but also those related to Colon Cancer, Cardiovascular disease and Alzheimer's disease [20].

Excess of macronutrients in the adipose tissues, stimulates them to release inflammatory mediators, such as tumor necrosis factor alpha, interleukin-6, and C-reactive protein [21]. Persistent subclinical inflammation predisposes to chronic diseases. Hence, the inflammatory pathway, seems to be a potential target for interventions, to reduce clinical complications related to obesity and aging [22,23]. Data from observational studies, show lower inflammatory biomarker concentrations in those, who report performing frequent and more intense physical activity $[18,22,23]$. The mechanisms by which increased physical activity, reduces persistent inflammation is not well understood. Yet another approach for the management of chronic inflammation seems to be dietary. As natural modulators of proinflammatory gene expressions, phytochemicals from fruits, vegetables and legumes could be incorporated into novel complementary anti-inflammatory formulations. The phenolics and triterpenoids in fruits and vegetables have been shown to possess high anti-inflammatory activity [24].

It gives me great pleasure in writing this editorial, for the Journal of Interventions in Obesity and Diabetes. In an earlier overview, we briefly discussed some management strategies for these metabolic diseases. As we have mentioned earlier, when it comes to the management of Obesity, we seem to have very limited choices, bariatric surgery, diet, and exercise. In this editorial, we have discussed interventions at the earliest possible stages of excess weight and obesity, even before birth and at early childhood. We also have discussed briefly two, early metabolic risks, oxidative stress and inflammation. We sincerely hope that the readers will consider these suggestions seriously and develop novel approaches to the management of these early metabolic risks.

\section{References}

1. NCD Risk Factor Task Force (NCD-RisC) (2016) Trends in body-mass index in 200 countries from 1975 to 2014: a pooled analysis of a698 population-based measurements with 19.2 million participants. Lancet 387(10026): 1377-1396

2. Ni Mhurchu C, Rodgers A, Pan WH, Gu DF, Woodward M (2004) Body mass index and cardiovascular disease in the asia-pacific region: an overview of 33 cohorts involving 310,000 participants. Int J Epidemiol 33(4): 751-758.

3. Bajaj H, Pereira M, Anjana M (2014) Comparison of relative waist circumference between Asian Indians. J of Obesity, p. 10.

4. De Gonzalez B, Hartge A, Cerhan JR, Flint AJ, Hannan L, et al. (2010) Body-mass index and mortality among 1,46 million white adults. N Engl J Med 363(23): 2211-2219.

5. Zheng W, McLerran DF, Rolland B, Zhang X, Inoue M, et al. (2011) Association between body-mass index and risk of death in more than 1 million Asians. N Engl J Med 364(8): 719-729.

6. Wormser D, Kaptoge S, Angelantonio E, Wood AM, Pennells L, et al. (2011) Separate and combined associations of body-mass index and abdominal adiposity with cardiovascular disease: collaborative analysis of 58 prospective studies. Lancet 377(9771): 1085-1095.

7. Whitlock G, Lewington S, Sherliker P, Clarke R, Emberson J, et al. (2009) Body-mass index and cause-specific mortality in 900, 000 adults: collaborative analyses of 57 prospective studies. Lancet 373(9669): 1083-1096.

8. Finucane MM, Stevens GA, Cowan MJ, Danaei G, Lin JK, et al. (2011) National, regional and global trends in body-mass index since 1980: systematic analysis of health examination surveys and epidemiological studies with 960 country-years and 9.1 million participants. Lancet 377(9765): 557-567.

9. Ng M, Fleming T, Robinson M, Thomson B, Graetz N, et al. (2014) Global, regional and national prevalence of overweight and obesity in children and adults during 1980-2013: a systematic analysis for the global burden of disease study 2013. Lancet 384: 766-781.

10. Stevens GA, Singh GM, Lu Y, Danaei G, Lin JK, et al. (2012) National, regional and global trends in adult overweight and obesity prevalences. Popular Health Metr 10(1): 22.

11. Cao Q, Yu S, Xiong W, Li Y, Li H, et al. (2018) Waist-hip ratio as a predictor of myocardial infraction risk: A systematic review and meta- analysis. Medicine (Baltimore) 97(30): e11639.

12. Mytton OT, Jackson C, Steinbacher A, Anna G, Claudia L, et al. (2018) The current and potential health benefits of the national health service health check cardiovascular disease prevention programmes in England: A microsimulation study. PLoS Med 15(3): e1002517.

13. Rao GHR (2018) Management of excess weight and obesity: A global perspective. Interventions Obes Daib 1(5).

14. Roy T (2013) Type-2 Diabetes: Etiology and reversibility. Diab Care 36(4): 1047-1055.

15. Barker DJ (2004) The developmental origins of adult disease. J Am Coll Nutr 23(suppl 6): 588S-595S.

16. Barker DJ (2005) The developmental origin of insulin resistance. Horm Res 64(Suppl 3): 2-7. 
17. Kawai K, Spiegelman D, Shankar AH, Fawzi WW (2010) Maternal multiple micronutrient supplementation and pregnancy outcomes in developing countries: meta-analysis and meta-regression. Bull World Health Organ 89(6): 402-411.

18. Kelly A, Wetzateon RJ, Kaiser DR, Steinberger J, Bank AJ, et al. (2004) Inflammation, insulin resistance and endothelial dysfunction in overweight children and adolescents. The J Pediatr 145(6): 731-736.

19. Marseglia L, Manti S, Angelo G (2015) Oxidative stress in obesity: A critical component in human diseases. Int J Mol Sci 16 (1): 378-400.

20. Hybertson BM, Gao B, Bose SK, McCord JM (2011) Oxidative stress in health and disease: The therapeutic potential of Nrf2 activation. Mol Aspects of Med 32(4-6): 234-246.
21. Ellulu MS, Patimah I, Khaza'ai H (2017) Obesity and inflammation: the linking mechanisms and the complications. Arch Med Sci 13(4): 851863.

22. Stephen C, Phillip C: Multiple interventions for treating chronic inflammation in older adults: What is the best way? Exerc. Sports Sci Rev 38(2): 99.

23. Nicklas BJ, Brinkley TE (2009) Exercise training as a treatment for chronic inflammation in the elderly. Exerc Sport Sci Rev 37(4): 165-170.

24.Zhu F, Du B, Xu B (2018) Anti-inflammatory effects of phytochemicals from fruits, vegetables, and food legumes: A review. Crit Rev Food Sci Nutr 58(8): 1260-1270.

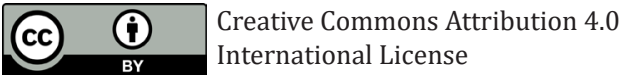

For possible submissions Click Here

\section{Submit Article}

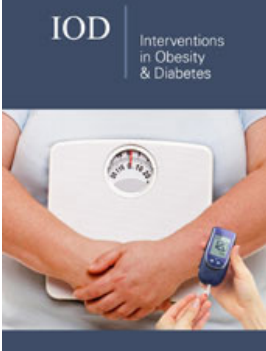

\section{Intervention in Obesity \& Diabetes}

\section{Benefits of Publishing with us}

- High-level peer review and editorial services

- Freely accessible online immediately upon publication

- Authors retain the copyright to their work

- Licensing it under a Creative Commons license

- Visibility through different online platforms 